\title{
Glucose, fructose and sucrose in minimally processed potato: Effect of cultivar, tuber storage time and heat treatment
}

\section{Abstract}

Recently, popularity of minimally processed potatoes (MPP) is growing as well as other minimally processed fruits and vegetables, due to its convenience and easy preparation, what consumers nowadays prefer. However, these types of products are very perishable, influenced by a number of factors, particularly cultivar and tuber condition. Potato is suitable for a long storage, but certain changes occur in its chemical composition during storage. These changes, among other factors, greatly depend upon cultivar, as well as storage conditions, and consequently affect on MPP durability and quality. Besides, MPP is subjected to heat treatment before consumption, where raw potatoes chemical composition and the way of heat treatment affect the chemical composition of the prepared potato dish. Potato sweetening is known phenomenon that occurs during storage, what negatively affect on health safety of fried potato.

Therefore, the aim of this study was to investigate the influence of cultivar and tubers storage time, as well as MPP storage time and heat treatment on the sugar content in raw, boiled and fried potatoes.

Potato cultivars Birgit and Lady Claire used in this study were stored for 1 and 4 months in warehouse $\left(8{ }^{\circ} \mathrm{C} / \mathrm{RH} 95 \%\right)$. MPP was produced by washing, peeling, slicing followed by anti-browning treatment (dipping in $2 \%$ sodium ascorbate solution/3 min), draining and vacuum packing in PE/PA bags. After $1^{\text {st }}, 5^{\text {th }}$ and $8^{\text {th }}$ day of storage at $10^{\circ} \mathrm{C}$, MPP were boiled and fried. All samples, including raw MPP, were freeze dried and sugar content was determined by UPLC MS/MS.

Most abundant sugar was sucrose, followed by glucose and fructose, where cv. Birgit contained slightly higher values of all sugars compared to cv. Lady Claire. Initial sugar content of both cultivars was very low. However, during tuber aging, as well as during MPP storage time, content of individual sugars increased in most of samples, with slightly higher increase in cv. Birgit raw samples. Furthermore, heat treatment affects on sugar composition, since glucose and fructose are generally reduced in boiled and fried potatoes with certain differences depending on the cultivar. Total sugar content of cv. Birgit MPP mostly increased during storage, regardless tuber age and the way of heat treatment, while in cv. Lady Claire samples opposite trend with few exceptions was observed. Generally, cv. Lady Claire has lower and slightly more stable sugar content upon tuber aging, MPP storage time and heat treatment.

\section{Conflict of Interest}

There is no conflict of interest 